\title{
Adaptation of the Adlerian Personality Priority Assessment into Turkish
}

\author{
Abdi Gungor (iD), ${ }^{*}$, Dalena Dillman Taylor (iD)2
}

${ }^{1}$ Guidance and Psychological Counseling Program, Düzce University, Düzce, Turkey

${ }^{2}$ Department of Counselor Education and School Psychology, University of Central Florida, Orlando, FL, USA

\section{ARTICLE HISTORY}

Received: Jan. 18, 2021

Revised: Sep. 14, 2021

Accepted: Oct. 25, 2021

Keywords:

Adlerian therapy,

The Adlerian personality priority assessment,

Adaptation,

Psychometric properties,

Reliability,

Validity.

\begin{abstract}
Personality priorities are important concepts in Adlerian theory, contributing to understanding and conceptualizing clients' lifestyles. Even though Adlerian psychology promises multicultural applications and has been interested in Turkey, no instrument measuring personality priorities has been developed or adapted into Turkish. Therefore, the purpose of this study was to adapt the Adlerian Personality Priority Assessment (APPA) into Turkish and examine its psychometric properties with a sample of Turkish undergraduate students. This study was conducted in three steps. In the first step, a linguistic equivalence test was performed with a sample of 73 students enrolled at the Department of English Language Education. In the second step, the structure of the APPA was examined using exploratory and confirmatory factor analyses with a sample of 1,279 undergraduate students. In the final step, test-retest reliability was tested with a sample of 93 undergraduate students within 4-week interval. The results of the linguistic equivalency study revealed that translations were linguistically and culturally proper. According to the exploratory and confirmatory factor analyses, the Turkish form of the APPA consisted of 24 items loaded with four factors consistent with the original form. The results also revealed good levels of internal and test-retest reliabilities. The findings of this study showed that the Turkish form of the APPA is a valid and reliable instrument, and it can be used in research and practice with Turkish populations. The results and limitations were discussed, along with implications for future research and practice.
\end{abstract}

\section{INTRODUCTION}

Adlerian theorists strongly consider individuals' social and cultural contexts when conceptualizing people within their social environments (Carlson \& Carlson, 2000). Individual psychology, created by Alfred Adler (1931), appears to be suitable for the characteristics of Turkish culture. For instance, Turkish culture values cooperation, connection with others, and social life, and the roles of family in early childhood are also highly emphasized in Turkish traditions (Sümer \& Rasmussen, 2012). In this regard, Adlerian psychology receives attention in Turkey for both practice and research. However, research within Turkish psychology practice appears to be in its infancy. For example, Adlerian concepts were conceptually discussed and reviewed in Turkish literature such as Adlerian encouragement within the counseling relationship (Ergün-Başak \& Ceyhan, 2011) and the use of Adlerian family counseling

\footnotetext{
*CONTACT: Abdi Gungor $\bowtie$ abdigungor@duzce.edu.tr 311, Merkez/Duzce 81620
} 
(Akçabozan \& Sümer, 2016). Those studies discussed the Adlerian concepts and provided suggestions for Adlerian practice in Turkish culture. Given the heightened interest in adopting Adlerian psychology in the Turkish culture (Sümer \& Rasmussen, 2012), it is crucial to develop valid and reliable instruments that measure the essential concepts of Adlerian theory, such as personality priorities.

\subsection{Individual Psychology}

Across all theoretical approaches, therapists work to conceptualize clients' presenting concerns and develop a treatment plan to intervene to best help clients overcome challenges. Within the Adlerian framework, the concept of understanding a client's presenting issue is positioned within their lifestyle: their view of self, others, and the world as influenced by genetic and environmental factors (Carlson et al., 2006). Although Adler (1929) stated, "we do not consider human beings types because every human being has an individual style of life" (p.102), he also noted that lifestyles serve as an intellectual device to understand similarities and differences between people. Even though Adlerian therapists prize each client's uniqueness and personal assets, understanding an overarching framework of individuals' lifestyles can be clinically useful (Kefir \& Corsini, 1974). In this regard, Kefir (1971) developed four personality priorities that capture broad categories of individuals' worldviews that can quickly provide the therapist insight into their lifestyle, mistaken beliefs/cognitive distortions, and how that worldview can impact their progress in therapy.

\subsection{Personality Priorities}

Kefir (1971) once described personality priorities as a "window into one's lifestyle," indicating this construct can be used as a snapshot into an individual's view of self, others, and the world. The brief snapshot can provide therapists with opportunities to present tentative hypotheses early on in the counseling process to begin deconstructing mistaken beliefs and developing more positive coping skills. However, Kefir and Corsini (1974) clarified that priorities are not considered fixed, providing fluidity for modifications in different situations (e.g., personal versus professional life). Priorities instead give insights on one's general dispositional set and central tendency towards life (Kefir \& Corsini, 1974). Each person functions from a primary priority, although they can constantly access all four as needed to strive for belonging and significance. Dependent upon early childhood experiences, individuals may choose to operate from their primary priority on the socially useful or useless side of life. This functionality allows the therapist to understand the level of discouragement or distress in which the client is currently presenting. Therefore, there is no hierarchy of priorities; each priority serves a purpose to enable the client to achieve their primary goals best.

Kefir (1981) originally defined four types of personality priorities: (a) avoider, (b) pleaser, (c) controller, and (d) morally superior. However, Pew (1976) modified the priorities as follows: control, pleasing, superiority, and comfort, removing the person's focus and replacing it with an action. This slight modification aligned more with the purpose of fluidity and appeared less trait-like. Due to page limitations, see Dillman Taylor et al. (2015) for complete definitions of the four priorities.

\subsection{Measuring Personality Priorities}

Dillman Taylor et al. (2015) developed the Adlerian Personality Priority Assessment (APPA) in response to the lack of validation of previous personality priority instruments (e.g., Allen Assessment for Adlerian Personality Priorities [AAAPP]; Langenfeld Inventory for Personality Priorities [LIPP]). The APPA was created to assess individuals' priority of achieving significance and belonging in their lives. The original study confirmed Kefir's (1971) four initial priorities with 393 undergraduates, concluding 30 items represented four personality priorities: control (six items), pleasing (nine items), superiority (seven items), and comfort 
(seven items). Dillman Taylor, Bratton, and Henson (2019) conducted another study to examine the psychometric properties of the APPA with a sample of 1201 undergraduate students. Results supported the four-factor structure of the APPA and provided preliminary results for the usefulness of the APPA for research and practice. For example, in a study utilizing the APPA to measure personality priorities, Dillman Taylor et al. (2018) examined the relationship between personality priorities and wellness in counselors-in-training and found that pleasing and comfort negatively predicted wellness even though superiority and control were not found significantly related to wellness. In addition, Dillman Taylor and Mullen (2019) modified the 30-item APPA to 22 items, resulting in a four-factor model: control (four items), pleasing (seven items), superiority (seven items), and comfort (four items). All studies examining the factorial structure of the APPA confirm four factors, which align with the original theoretical structure of personality priorities. Therefore, preliminary evidence of the internal structure of the APPA, using EFAs and confirmatory factor analyses across studies, was demonstrated in addition to evidence of relationship to other variables (e.g., wellness, Dillman Taylor et al., 2018).

Even though the studies on Adlerian concepts in Tutkish culture were limited, there is a grwoing interest (e.g., Akçabozan \& Sümer, 2016; Ergün-Başak \& Ceyhan, 2011; Sümer \& Rasmussen, 2012). As discussed, understanding personality priorities contribute to conceptualizing the person's lifestyle (Kefir, 1971; Kefir \& Corsini, 1974). To date, an instrument assessing personality priority in Turkey does not exist. However, validated instruments are needed to carry out further research on Adlerian theory and practice. Thus, the purpose of this study was to adapt the APPA to Turkish culture, specifically focusing on the internal structure. More specifically, this study aimed to examine the reliability and validity of the APPA with Turkish undergraduate students.

\section{METHOD}

\subsection{Data Collection}

A convenient sampling method was used in this study. We received approval from the university ethical board in Turkey to conduct this study. Based on outlined data collection procedures, we followed university protocol to obtain permission from dean offices and class instructors to recruit participants. All data were collected in person via paper-and-pencil. We followed detailed procedures to ensure confidentiality and that participation was voluntary. For this study, we conducted a three-part process in data collection. For the first part of the study, 73 participants completed both the English and Turkish forms of the APPA for the linguistic equivalence within a 2-week interval. Based on the results of the linguistic equivalence test, translations of four items were revised. Once the translation process was finalized, we collected data from the second group $(\mathrm{n}=1279)$. Finally, the Turkish form of the APPA was implemented twice to the last group $(n=93)$ for test-retest reliability within a 4-week interval.

\subsection{Participants}

A total of 1445 undergraduate students who attended a public university in the northwest of Turkey participated in this study. This study consisted of three independent samples. In the first sample, 73 students enrolled at the Department of English Language Education participated in the linguistic equivalence study. To qualify for part one of this study, the participants needed to demonstrate fluency in both Turkish and English. This qualification was to ensure the accuracy of responses to items of the English and the Turkish version of the APPA.

In the second sample, 1279 undergraduate students, whose age ranged from 17 to $38(M=$ $20.81, S D=2.46$ ) participated. To qualify for part two of this study, the participants attended a four-year undergraduate program. The data of this group was randomly split into two equal subsamples $\left(n_{1}=n_{2}=639\right)$; one case was randomly removed to ensure equal sample sizes. Age 
ranged from 17 to $38(M=20.79, S D=2.43)$ in the subsample 1 , and from 17 to $38(M=20.83$, $S D=2.49$ ) in the subsample 2. Table 1 shows demographic characteristics of the participants, such as gender, grade, and birth order for the total sample for part two, subsample 1, and subsample 2. In addition, we asked participants to rate their perceived socioeconomic status. As shown in Table 1, the total sample for part two of this study mostly reflected middle-class socioeconomic status.

Table 1. Comparison Demographics of total group subsample groups

\begin{tabular}{lccc}
\hline \multicolumn{1}{c}{ Demographic } & Part II Total Sample $n(\%)$ & $\begin{array}{c}\text { Subsample } 1 n \\
(\%)\end{array}$ & $\begin{array}{c}\text { Subsample } 2 n \\
(\%)\end{array}$ \\
\hline Gender & & & \\
Male & $305(23.8)$ & $153(23.9)$ & $152(23.8)$ \\
Female & $958(74.9)$ & $477(74.7)$ & $480(75.1)$ \\
Missing & $16(1.3)$ & $9(1.4)$ & $7(1.1)$ \\
Grade & & & \\
Freshman & $449(35.1)$ & $216(33.8)$ & $233(36.5)$ \\
Sophomore & $214(16.7)$ & $116(18.2)$ & $98(15.3)$ \\
Junior & $236(18.5)$ & $116(18.2)$ & $120(18.8)$ \\
Senior & $375(29.3)$ & $188(29.4)$ & $186(29.1)$ \\
Missing & $5(.4)$ & $3(.4)$ & $2(.3)$ \\
Birth order & & & \\
First & $460(36)$ & $214(33.5)$ & $245(38.3)$ \\
Second/middle & $526(41.1)$ & $264(41.3)$ & $262(41)$ \\
Last & $246(19.2)$ & $135(21.1)$ & $111(17.4)$ \\
Only & $32(2.5)$ & $17(2.7)$ & $15(2.4)$ \\
Missing & $15(1.2)$ & $9(1.4)$ & $6(.9)$ \\
Perceived economic status & & & \\
High & $58(4.5)$ & $33(5.2)$ & $25(3.9)$ \\
Middle & $1147(89.7)$ & $563(88.1)$ & $583(91.2)$ \\
Low & $59(4.6)$ & $33(5.2)$ & $26(4.1)$ \\
Missing & $15(1.2)$ & $10(1.5)$ & $5(.8)$ \\
\hline
\end{tabular}

For the test-retest reliability study (part 3), 93 participants (17 males, 76 females) completed the APPA. Ages in this group ranged from 20 to $35(M=23.17, S D=2.81)$. To qualify, participants in this group needed to be undergraduate students who attended a four-year program.

\subsection{Measurements}

\subsubsection{The Adlerian Personality Priority Assessment (APPA)}

Dillman Taylor et al. (2015) developed the APPA to measure Adlerian personality priorities as a mechanism to assess mistaken beliefs or cognitive distortions, which were originally proposed by Kefir (1971). The APPA has 30 items measuring four personality priorities: control (six items), pleasing (nine items), superiority (seven items), and comfort (seven items). Items are on a 5-point Likert scale ranging from not at all (1) to very much (5). A higher score of a particular personality priority indicates that a person views the world more in line with the characteristics of that priority to achieve significance and belonging in their life. Sample items include "In most situations, I prefer to be in charge" (control), "I need to know that others are pleased with me" (pleasing), "I need to be the winner in games" (superiority), and "I prefer not having a lot of work to do" (comfort). 
Dillman Taylor et al. (2015) reported that the full model reproduced $47.29 \%$ of the variance. Each factor explained for each factor was $16.93 \%$ for pleasing, $10.57 \%$ for control, $10.13 \%$ for comfort, and $9.66 \%$ for superiority. In addition, the following studies confirmed the four-factor structure of the APPA with samples of 1,210 undergraduate students (Dillman Taylor et al., 2019) and 1,019 adults (Dillman Taylor \& Mullen, 2019). Dillman Taylor et al. (2015) reported Cronbach Alpha coefficients for each factor as follows: .91 for pleasing, .80 for comfort, .81 for control, and .88 for superiority. The current study reported Cronbach Alpha coefficients for each factor as following .84 for superiority, .81 for pleasing, .68 for comfort, and .78 for control.

\subsubsection{Translation procedure}

We conducted the translation process of the APPA into Turkish using the five-step model as suggested to adapt an original instrument into another language and culture (e.g., Abubakar et al., 2013; Carlson, 2000). First, four faculty members in counseling departments who were native Turkish speakers and had fluent English separately translated the items of the APPA into Turkish. Second, all translations were compared and analyzed, and the most accurate translations were chosen for each item. Third, a faculty member in Teaching English to Speakers of Other Languages department in the United States, who was a native Turkish speaker and unfamiliar to the APPA, reverse translated the items into English. Previous researchers have suggested reverse translation as a part of the adaption procedure to ensure the correctness of the translation (Abubakar et al., 2013; Geisinger, 1994). Fourth, two faculty members in counselor education in the United States, who were highly familiar with Adlerian theory, compared the back-translated items with the original APPA in terms of correctness, clarity, and cultural relevancy. Finally, the reverse-translated version of the APPA was reviewed and approved by the developer of the original form of the APPA. In line with the original scale, the final version of the APPA-Turkish (APPA-T) contains 30 items on a 5-point Likert scale $(1=$ Not at all, $2=$ A little bit, $3=$ Somewhat, $4=$ Quite a bit, and $5=$ Very much $)$. In the final step, a translation equivalency test was implemented with 73 undergraduate students enrolled at the Department of English Language Education in Turkey. Based on the translation equivalency test results, two faculty members in counselor education revised four items, thus finalizing the APPA-T.

\subsection{Data Analysis}

We conducted analyses using Statistical Package for the Social Sciences (SPSS) version 22.0 and Analysis of Moment Structures (AMOS) version 23. Before data analysis, a data screening procedure was employed. More specifically, encoding data, missing data, and outliers were checked. In addition, we examined normality, multicollinearity, and missing data. Results are presented in the following sections. In study 1 , we employed a translation equivalency study to investigate the accuracy of the translation.

In study 2, we examined the structure of the original APPA using confirmatory factor analysis (CFA), but the results revealed a poor-fitting model. Thus, we tested the dimensionality of the APPA with this Turkish sample. As recommended for cross-validation (Gerbing \& Hamilton, 1996), we randomly split the sample $(N=1279)$ into two subsamples $\left(n_{1}=639, n_{2}=639\right)$ using the random sample selection procedure in SPSS 22.0, and randomly deleted one participant to obtain equal samples. Then, we conducted exploratory factor analysis (EFA) to determine the factor structure of the APPA-T with subsample 1. Next, a CFA was conducted with subsample 2 to confirm the structure acquired as the result of the EFA. Cronbach's alpha coefficients of each factor were estimated with the whole sample to examine the internal consistency reliability of the APPA-T. In study 3, we conducted a test-retest study with 93 students within a 4-week interval. 


\section{RESULT / FINDINGS}

Regarding preliminary analyses, we checked all assumptions for the data analyses employed across the three studies. First, we employed Little's MCAR tests to examine whether the data sets were completely at random. For all three subsamples, data were found to be at random (Study 1, $x^{2}=857.03, d f=878, p>.05$; Study 2, $x^{2}=1027.99, d f=968, p>.05$; Study $3, x^{2}=$ $479.18, d f=472, p>.05)$. We checked all assumptions, including $\mathrm{z}$ scores for potential outliers, kurtosis and skewness for normality, and multicollinearity (Tabachnick \& Fidell, 2013). For all three studies, assumptions were met, thus demonstrating that the data is appropriate for the selected analyses.

\subsection{Study 1: Translation Equivalency Test}

In an adaption of an instrument into another language and culture, Carlson (2000) suggested three steps for the translation process: (1) one-way translation is conducted by bilingual experts translating the original instrument into the target language; (2) back-translation method is performed as an independent bilingual expert translates back the target-language version into the original language; and (3) a translation equivalency test is conducted to ensure a culturally equivalent translation. In preparation for this study, we conducted the first two steps prior to implementing with participants, as noted previously. Therefore, we asked 73 bilingual undergraduate students to complete the original 30-item APPA first then the APPA-T within a 2-week interval. The main purpose of an equivalency test is to utilize two forms of an instrument into the same group and compare the results. If the translations are deemed accurate and meaningful, no difference between the two implementations is expected (Carlson, 2000; Hambleton et al., 2004). Thus, we conducted paired-samples t-tests to compare the participants' scores on the two different versions of the APPA (Pallant, 2010). The results showed that there were no statistically significant differences in mean scores of two implementations for each of the priorities: superiority $(t(51)=.29, p=.77)$, pleasing $(t(51)=-1.46, p=.15)$, comfort $(t(51)$ $=.55, p=.59)$, and control $(t(51)=.99, p=.32)$. Therefore, these findings supported the translation equivalency between the APPA and the APPA-T.

For further analysis, we inspected the results of both assessments (e.g., APPA and APPA-T) to ensure the accuracy of translations. Due to the ordinal nature of the items ranging from one to five, we conducted the Wilcoxon matched pairs signed ranks test for each item across the two assessments (Pallant, 2010; Tabachnick \& Fidell, 2013). The results indicated only four items (APPA Item 5, 8, 18, and 24) had statistically significant differences between scores across the two instruments. These findings support the accuracy of the translation for the majority of the items. Further, we examined correlations between the items in English and Turkish using the Spearman rho formula (Pallant, 2010; Tabachnick \& Fidell, 2013). All results, including mean ranks for each item, are reported in Table 2. The results revealed that most correlations between English and Turkish items were statistically significant, except for three items (APPA Item 2, 17, and 28). Therefore, as suggested (Carlson, 2000), we inspected these seven items; two Turkish faculty revised these items in terms of clarity and cultural relevancy. 
Table 2. Wilcoxon Matched Pairs Signed Ranks Test, Correlations, Means, and Standard Deviations for Each Item.

\begin{tabular}{|c|c|c|c|c|}
\hline \multirow{2}{*}{ Items } & APPA & APPA-T & \multirow{2}{*}{ rho } & \multirow{2}{*}{$Z$} \\
\hline & $M(S D)$ & $M(S D)$ & & \\
\hline APPA Item 1 & $2.81(1.10)$ & $2.72(.98)$ & $.31 *$ & -.99 \\
\hline APPA Item 2 & $2.79(1.27)$ & $2.36(.97)$ & .24 & -1.44 \\
\hline APPA Item 3 & $3.33(1.31)$ & $3.70(1.15)$ & $.51 * *$ & -1.19 \\
\hline APPA Item 4 & $3.12(1.14)$ & $3.35(.95)$ & $.56^{* *}$ & -1.81 \\
\hline APPA Item 5 & $2.67(1.38)$ & $2.37(1.33)$ & $.69 * *$ & $-2.74 * *$ \\
\hline APPA Item 6 & $2.77(1.16)$ & $3.10(1.13)$ & $.60 * *$ & -.31 \\
\hline APPA Item 7 & $2.64(1.23)$ & $2.68(.99)$ & $.43^{* *}$ & -.01 \\
\hline APPA Item 8 & $2.52(1.31)$ & $3.02(1.46)$ & $.59 * *$ & $-2.37 *$ \\
\hline APPA Item 9 & $3.32(1.23)$ & $3.36(1.10)$ & $.39 * *$ & -2.35 \\
\hline APPA Item 10 & $3.07(1.36)$ & $3.40(1.06)$ & $.46^{* *}$ & -1.87 \\
\hline APPA Item 11 & $2.68(1.33)$ & $2.53(1.24)$ & $.55^{* *}$ & -1.35 \\
\hline APPA Item 12 & $3.42(1.09)$ & $3.66(.94)$ & $.31 *$ & -.97 \\
\hline APPA Item 13 & $3.41(1.07)$ & $3.47(.87)$ & $.36^{* *}$ & -.06 \\
\hline APPA Item 14 & $3.31(1.29)$ & $3.38(1.14)$ & $.74 * *$ & -.36 \\
\hline APPA Item 15 & $3.35(1.21)$ & $3.39(1.18)$ & $.38 * *$ & -.89 \\
\hline APPA Item 16 & $3.34(1.26)$ & $3.17(1.21)$ & $.55^{* *}$ & -.85 \\
\hline APPA Item 17 & $3.04(1.04)$ & $3.42(1.24)$ & .20 & -1.83 \\
\hline APPA Item 18 & $2.13(1.16)$ & $1.69(.93)$ & $.46^{* *}$ & $-2.41 *$ \\
\hline APPA Item 19 & $3.19(1.31)$ & $3.33(1.14)$ & $.51^{* *}$ & -.49 \\
\hline APPA Item 20 & $3.01(1.25)$ & $2.98(1.29)$ & $.63 * *$ & -.21 \\
\hline APPA Item 21 & $2.88(1.26)$ & $2.95(1.23)$ & $.65^{* *}$ & -.89 \\
\hline APPA Item 22 & $3.60(1.28)$ & $3.65(1.10)$ & $.60^{* *}$ & -.07 \\
\hline APPA Item 23 & $3.09(1.80)$ & $2.85(1.18)$ & $.45^{* *}$ & -1.87 \\
\hline APPA Item 24 & $3.07(1.24)$ & $2.42(1.09)$ & $.50 * *$ & $-3.74 * * *$ \\
\hline APPA Item 25 & $3.11(1.35)$ & $3.02(1.33)$ & $.54^{* *}$ & -.69 \\
\hline APPA Item 26 & $3.07(1.09)$ & $3.30(1.06)$ & $.57 * *$ & -1.84 \\
\hline APPA Item 27 & $2.67(1.13)$ & $2.63(1.26)$ & $.61^{* *}$ & -.55 \\
\hline APPA Item 28 & $2.27(1.07)$ & $2.13(.03)$ & .19 & -1.27 \\
\hline APPA Item 29 & $2.97(1.29)$ & $2.98(1.14)$ & $.61 * *$ & -.68 \\
\hline APPA Item 30 & $3.21(1.27)$ & $3.12(1.25)$ & $.68 * *$ & -1.17 \\
\hline
\end{tabular}

Note. $* p<.05, * * p<.01, * * * p<.001$

\subsection{Study 2: Structure Analyses}

\subsubsection{Confirmatory factor analysis}

We conducted a CFA to test the original structure of the APPA developed by Dillman Taylor et al. (2015) with the entire sample $(N=1279)$. To evaluate the model, we used the following fit indices to determine the overall goodness of the model: (a) chi-square ratio $(<.4)$, (b) comparative fit index (CFI, $>.94)$, (c) goodness of fit (GFI, $>.90)$, root mean square error of approximation (RMSEA, < .09), and standardized root mean square residual (SRMR, < .06; Brown, 2014; Hooper et al., 2008; Hu \& Bentler, 1999; Kline, 2011; O'Rourke et al., 2013; Weston \& Gore, 2006). The CFA results indicated a poor fit for the original APPA: $x^{2}(d f=$ $399, N=1279)=2,501.01, p<.001 ; x^{2} / d f=6.27$; CFI $=.82$; GFI $=.87$; RMSEA $=.06$; SRMR $=.08$. Therefore, this model was deemed to be insufficient for the current data. Because the original factor structure of the APPA was not supported with this Turkish sample, as suggested 
(Gerbing \& Hamilton, 1996), we reexamined the dimensionality of the APPA with this Turkish sample. Thus, we randomly split the data into two equal subsamples: Subsample $1\left(n_{l}=639\right)$ and subsample 2 subsample $\left(n_{2}=639\right)$.

\subsubsection{Exploratory factor analysis}

To determine the factor structure of the Turkish version APPA, with subsample $1\left(n_{1}=639\right)$, we conducted a principal component analysis (PCA; Pallant, 2010) with direct varimax rotation because factors were not expected to be theoretically correlated (Costello \& Osborne, 2005; Pallant, 2010). The item-to-case ratio was 1:21, ideal for this analysis (Costello \& Osborne, 2005). In addition, regarding the suitability of the sample for factor analysis, we met the assumptions for Bartlett's test of sphericity $\left(x^{2}=6253.34, d f=435, p<.001\right)$ and Kaiser-MeyerOlkin (KMO = .86; Hair et al., 2010; Mvududu \& Sink, 2013). These results indicated that the data was appropriate for the use of factor analysis.

In order to determine the number of factors to retain, we inspected Eigenvalues higher than 1, the scree plot, and conducted parallel analysis (Pallant, 2010; Tabachnick \& Fidell, 2013). The results of the principal component analysis with varimax rotation found six factors with Eigenvalues greater than 1. However, when we inspected the scree plot, a significant break occurred between the fourth and fifth factors, which suggested a four-factor structure. Results from the parallel analysis also suggested retaining four factors. Thus, we retained four factors for the initial factor analysis. One item demonstrating significant cross-loading ( $>.32$; Costello \& Osborne, 2005; Tabachnick \& Fidell, 2013) was removed from the model. We reran the model to determine model fit with 29 items. Further, we found that items 15 and 18 on the APPA-T loaded on a different factor than anticipated (Dillman Taylor et al., 2015); however, we elected to retain it based on theoretical support.

The final model (see Table 3) comprised of a four-factor structure with 29 items, which were consistent with the original model: Factor 1 (superiority, nine items), Factor 2 (pleasing, nine items), Factor 3 (comfort, seven items), and Factor 4 (control, four items). The 4-factor model explained $48.21 \%$ of the total variance, appropriate for social sciences (Hair et al., 2010; Mvududu \& Sink, 2013).

Table 3. Factor Pattern/Factor Loadings for Exploratory Factor Analysis with Varimax Rotation of $A P P A-T$

\begin{tabular}{lcccc}
\hline Items & $\begin{array}{c}\text { Factor } 1 \\
\text { Superiority }\end{array}$ & $\begin{array}{c}\text { Factor } 2 \\
\text { Pleasing }\end{array}$ & $\begin{array}{c}\text { Factor } 3 \\
\text { Comfort }\end{array}$ & $\begin{array}{c}\text { Factor } 4 \\
\text { Control }\end{array}$ \\
\hline APPA Item 20 & $\mathbf{. 8 4}$ & .15 & -.13 & .03 \\
APPA Item 19 & $\mathbf{. 8 1}$ & .12 & -.09 & .06 \\
APPA Item 30 & $\mathbf{. 7}$ & .04 & -.03 & .14 \\
APPA Item 16 & $\mathbf{. 7 1}$ & .05 & .14 & .02 \\
APPA Item 25 & $\mathbf{. 7 0}$ & .12 & -.09 & .15 \\
APPA Item 14 & $\mathbf{. 6 0}$ & .20 & -.20 & .17 \\
APPA Item 26 & $\mathbf{. 5 6}$ & .04 & .31 & .14 \\
APPA Item 15 & $\mathbf{. 5 5}$ & .26 & .21 & .08 \\
APPA Item 18 & $\mathbf{. 4 2}$ & -.10 & .21 & .10 \\
APPA Item 27 & .06 & $\mathbf{. 7 4}$ & .09 & .16 \\
APPA Item 17 & .12 & $\mathbf{. 7 0}$ & -.09 & .03 \\
APPA Item 11 & .10 & $\mathbf{. 6 9}$ & -.03 & .18 \\
APPA Item 21 & .21 & $\mathbf{. 6 9}$ & .01 & .02 \\
APPA Item 3 & -.13 & $\mathbf{. 6 8}$ & -.06 & .03 \\
\hline
\end{tabular}


Table 3. Continues

\begin{tabular}{|c|c|c|c|c|}
\hline APPA Item 10 & .27 & .67 & .09 & .05 \\
\hline APPA Item 5 & .03 & .58 & .08 & -.14 \\
\hline APPA Item 8 & -.02 & .53 & .18 & .06 \\
\hline APPA Item 13 & .23 & .53 & .17 & .14 \\
\hline APPA Item 6 & -.02 & .00 & .71 & -.19 \\
\hline APPA Item 7 & .03 & .05 & .61 & -.21 \\
\hline APPA Item 29 & .05 & .14 & .58 & -.25 \\
\hline APPA Item 28 & .07 & .08 & .56 & .05 \\
\hline APPA Item 4 & .00 & .12 & .54 & -.32 \\
\hline APPA Item 22 & .12 & -.13 & .45 & .04 \\
\hline APPA Item 2 & -.18 & .18 & .43 & .00 \\
\hline APPA Item 23 & .19 & .13 & -.13 & .82 \\
\hline APPA Item 24 & .24 & -.02 & -.04 & .73 \\
\hline APPA Item 12 & .14 & .16 & -.17 & .71 \\
\hline APPA Item 9 & .12 & .19 & -.29 & .70 \\
\hline$\%$ variance & 20.78 & 12.47 & 10.13 & 4.83 \\
\hline Eigenvalue & 6.03 & 3.62 & 2.94 & 1.40 \\
\hline
\end{tabular}

\subsubsection{Second confirmatory factor analysis}

The next step of the investigation of structural validity of the APPA-T was conducting a CFA with subsample $2\left(n_{2}=639\right)$, a unique sample from subsample 1 used in the EFA. We conducted a CFA on the model derived as the result of the EFA, which indicated a four-factor structure with 29 APPA-T items. The model revealed a poor fit across some indices: $x^{2}(d f=371, N=$ $639)=1172.38, p<.001 ; x^{2} / d f=3.16 ; \mathrm{CFI}=.84 ; \mathrm{GFI}=.88 ; \mathrm{RMSEA}=.06$; $\mathrm{SRMR}=.06$. Fit indices produced mixed results, so we inspected the modification indices to improve the model.

Based on modification indices, we systematically removed APPA items 5, 15, and 16 due to covarying not being theoretically justified. In addition, we removed items 18 and 22 from the model because the loading of that items was lower than .30 (Mvududu \& Sink, 2013). Due to theoretical support, we elected to free errors between APPA items 10-13 and 23-24. Items 10 and 13 relate to one's desire to know that others are pleased with them; whereas, items 23 and 24 convey an individual's preference of being in charge.

With these modifications, the final model resulted in a 24 -item, four-factor structure, which also produced a mixed outcome with some indices demonstrating good fit while others show adequate fit: $x^{2}(d f=244, N=639)=649.21, p<.001 ; x^{2} / d f=2.66$; CFI $=.90$; GFI $=.92$; RMSEA $=.05 ;$ SRMR $=.06$. The standardized regression weights revealed that the loadings for all items were statistically significant, and ranged from .36 to .82, (Figure 1). In addition, the CFA revealed various relationships among priorities/factors. The superiority factor was found to be statistically correlated to pleasing $(r=.31, p<.001)$ and control $(r=.34, p<.001)$; and control was found to be statistically correlated to pleasing $(r=.36, p<.001)$ and comfort $(r=-.49, p<.001)$. Finally, we ran Cronbach's alpha internal consistency with the whole sample $(N=1279)$ for the final modified model derived from the second CFA and the found the following: .84 for superiority, .81 for pleasing, .69 for comfort, and .78 for control. 
Figure 1. CFA Diagram of the Final Modified Model for the APPA-T.

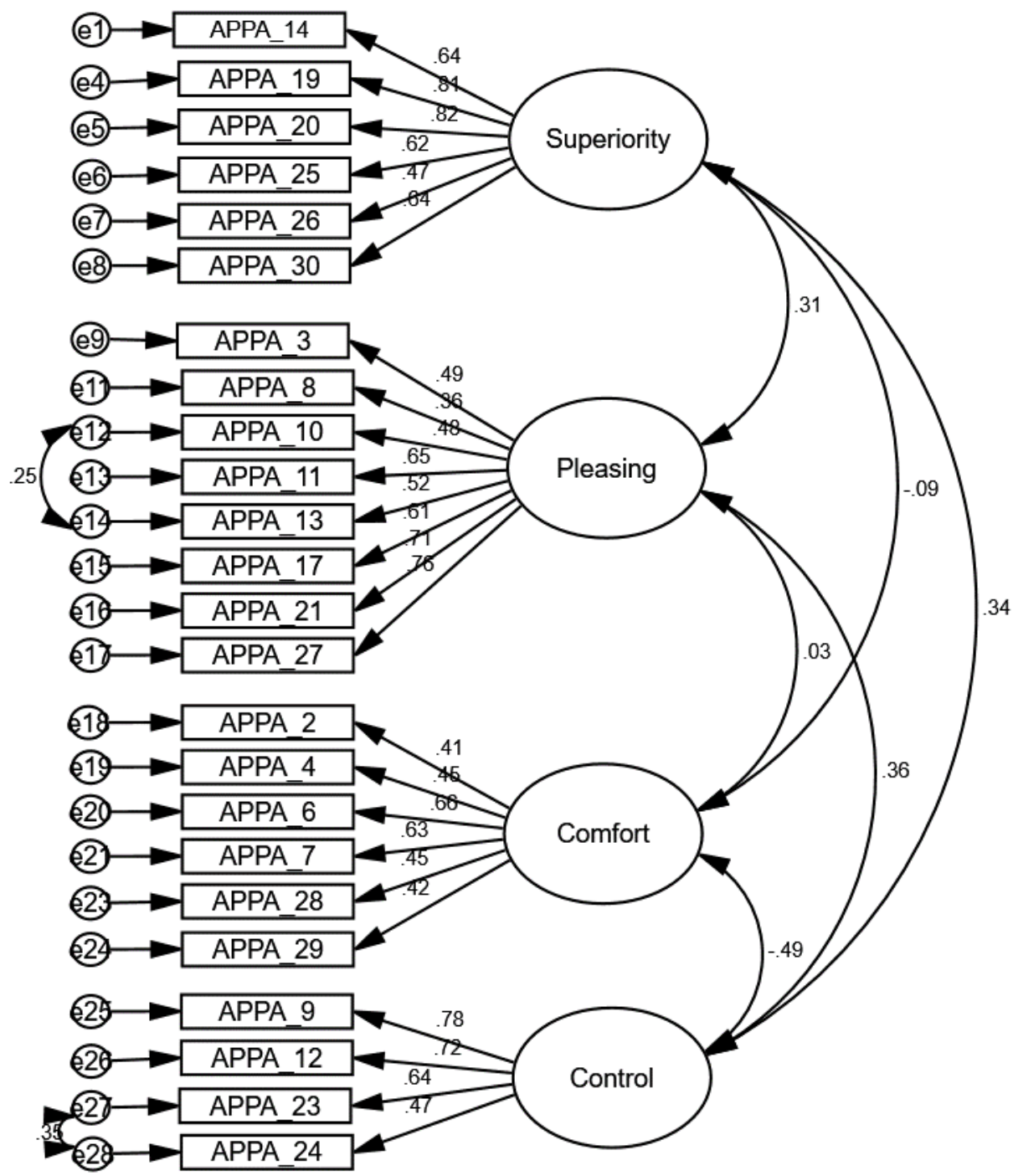

\subsection{Study 3: Test-Retest Reliability Analysis}

To determine the test-retest reliability, we administered the APPA-T to 93 undergraduate students within 4-week interval. The correlation coefficients between two applications were all significant: $(r=.72, p<.01)$ for superiority, $(r=.85, p<.01)$ for pleasing, $(r=.73, p<.01)$ for comfort, and $(r=.79, p<.01)$ for control. Thus, those results showed that the APPA-T is a reliable instrument.

\section{DISCUSSION and CONCLUSION}

Personality priorities are important variables in Adlerian theory to understand individuals' worldviews and relate to others. The concept of personality priorities provides insight into individuals' goals in which they strive for significance and belonging (Dillman Taylor et al., 2015; Kefir, 1971; Kefir \& Corsini, 1974). Kefir (1981) first introduced the concept of personality priorities. Shortly after that, Langenfeld and Main (1983) developed the first instrument, the LIPP, to measure personality priorities, although the items on the instrument presented with limited reliability (Ashby et al., 1998; Ashby et al., 2006; Dillman Taylor et al., 2015). Dillman Taylor et al. (2015) developed the APPA measuring personality priorities with a four-factor structure to address these concerns. Several studies demonstrate adequate to strong psychometric properties of the APPA (Dillman Taylor \& Mullen 2019; Dillman Taylor et al. 
2015; Dillman Taylor et al. 2019). Therefore, the researchers sought in this manuscript to adapt this instrument for the Turkish population.

We conducted three studies in order to establish the credibility of the revised instrument. First, we followed stringent guidelines for translating the APPA to the APPA-T form (Abubakar et al., 2013; Carlson, 2000) and conducted a translation equivalency test to confirm the adequate translation. Further, we employed an EFA and a CFA to examine the structural validity of the APPA-T. Regarding reliability, we estimated Cronbach's alpha coefficients to determine internal consistency and conducted a test-retest to measure the stability of the APPA-T over time. Overall, we found that the translation of APPA-T was linguistically and culturally appropriate for the undergraduate population in which we tested the instrument.

\subsection{Factorial Structure}

The original version of the APPA included four factors with 30 items with an undergraduate student sample (Dillman Taylor et al., 2015). Consistent with the previous studies (e.g., Dillman Taylor et al., 2019; Dillman Taylor \& Mullen, 2019), the current results of the EFA produced a 4-factor structure for the APPA-T, and the CFA confirmed this structure with a sample of undergraduate students. However, unlike the original version with 30 items, the APPA-T included 24 items because we deleted APPA items 1, 5, 15, 16, 18, and 22 from the model due to poor loadings or substantial cross-loadings. These findings are consistent with previous studies that removed items as well (e.g., Dillman Taylor et al., 2019; Dillman Taylor \& Mullen, 2019). However, the current study, similar to previous studies, removed items, all studies found a similar four-factor structure, inclusive of the personality priorities superiority, pleasing, control, and comfort. Hence, this study supports that the factorial structure of the APPA-T is valid for Turkish undergraduate students. This finding also indicates that the four personality priorities, proposed by Kefir (1971) as one of the concepts of Adlerian theory, can be applicable for Turkish culture. At least theoretically, this result suggests that Adlerian personality priorities are multiculturally sensitive to Turkish culture and a possible mechanism to view Turkish individuals' view of self, others, and the world.

\subsection{Implication for Counseling Practice}

The preliminary results of this study found that the APPA-T appears to be an applicable and valid instrument for the Turkish undergraduate sample. The APPA measures four personality priorities, which provides insight into individuals' lifestyle or worldview (Kefir, 1971; Ward, 1979). Understanding a client's lifestyle is one of the crucial goals in Adlerian therapy. More specifically, Adlerian psychotherapy identifies four steps: (1) establishing the therapeutic relationship, (2) assessing and understanding the lifestyle, (3) gaining insight, and (4) reeducation or reorientation (Oberst \& Stewart, 2003; Sweeney, 2009). In the second phase, the role of a therapist is to understand and conceptualize client's lifestyle to gain insight into their presenting problems to aid in the development of a treatment plan. Adlerian theorists provided several ways to understand clients' lifestyles, such as family constellation, family values, birth order, early collections, and personality priorities (Ansbacher \& Ansbacher, 1956; Sweeney, 2009). In addition, formalized lifestyle assessment tools such as Basic Adlerian Scales for Interpersonal Success-Adult Form (BASIS-A; Kern et al., 1997) and the APPA (Dillman Taylor et al., 2015) have been suggested to gather information about lifestyle (Dillman Taylor \& Mullen, 2019; Oberst \& Stewart, 2003). The BASIS-A is a more in-depth instrument to examine a client's lifestyle. On the other hand, the APPA help therapists briefly assess goals of significance and belonging to develop tentative hypotheses to help clients understand what behaviors, thoughts and/or feelings are keeping them stuck. In this sense, Turkish counseling professionals can use the APPA-T in their practice to guide their conceptualization and treatment of their clients' lifestyles. However, it should also be noted that Adlerian therapists 
tend to avoid over-categorizing individuals into types by asserting that each person is unique in his or her context (Adler, 1929). Therefore, we suggest using the APPA-T as a conversation starter to provide tentative hypotheses regarding the clients' lifestyle in the therapeutic work. For example, practitioners in Turkey can utilize APPA-T to their clients, especially during the initial phase of the therapy process, to gather initial information of presenting problems, which can relate to clients' lifestyles. This is especially crucial when considering the lack of an instrument in Turkish to measure clients' lifestyles.

\subsection{Limitations and Future Research}

This study examined the psychometric properties and provided evidence of the use of the APPA-T with a sample of Turkish undergraduate students. Even though the study sample met the requirements of conducting the EFA and CFA, one of the study's limitations is that the sample consisted of only Turkish undergraduate students, representing a non-clinical population, limiting the generalizability to non-clinical samples. However, the preliminary results from this study indicate the possibility of the APPA-T as a possible option. Therefore, the researchers believe that the APPA-T should be evaluated with a clinical sample in future research. University students also represent a young and educated population when compared to the rest of society. Therefore, future studies should investigate the validity and reliability of the APPA-T with a variety of other Turkish samples (e.g., clinical populations; various educational, socioeconomic, and age groupings).

Another limitation is that the researchers confirmed the APPA-T with 24 items instead of the original 30-item APPA. Nevertheless, this study found a four-factor structure consistent with the original model (Dillman Taylor et al., 2015) and previous findings (Dillman Taylor \& Mullen, 2019; Dillman Taylor et al., 2019). Future studies can be helpful to retest the factorial structure with 30-item and 24-item APPA-T with various other Turkish samples to confirm the findings of this study. Although this study investigated the construct validity of the APPA-T with Turkish undergraduate students, future studies are also recommended to examine the divergent and convergent validity of the APPA-T with other constructs such as other Adlerian concepts (e.g., social interest, feeling of inferiority, life style), concepts from other theories (e.g. cognitive schemas from cognitive theory), and/or other personality measures (e.g., NEO personality inventory- revised, MBTI).

\subsection{Conclusion}

Understanding one's lifestyle is crucial in Adlerian therapy, and personality priorities were developed to quickly and efficiently assess how individuals view self, others, and the world to treat clients in a more timely manner (Kefir, 1971). Dillman Taylor et al. (2015) developed the APPA to assess individuals' primary personality priority. The researchers adapted the APPA into Turkish and tested its psychometric properties with a Turkish sample in this study. The preliminary results revealed that the items on the APPA-T are reliable and valid for use in research and practice with Turkish undergraduate students. In addition, the results of this study supported that Adlerian personality priorities are appropriate for Turkish culture, which is consistent with multicultural aspects of Adlerian theory. Nonetheless, future studies would be helpful to continue testing the APPA-T with Turkish samples.

\section{Declaration of Conflicting Interests and Ethics}

The authors declare no conflict of interest. This research study complies with research publishing ethics. The scientific and legal responsibility for manuscripts published in IJATE belongs to the authors. Ethics Committee Number: Düzce University, 29/06/2018-2018/24. 


\section{Authorship Contribution Statement}

Abdi GUNGOR: Research design, literature review, data collection, data analysis, methodology, software, resources, discussion, writing. Dalena DILLMAN TAYLOR: Research design, supervision, validation, methodology, discussion, writing.

\section{ORCID}

\section{Abdi Gungor (iD https://orcid.org/0000-0002-7945-0906}

Dalena Dillman Taylor (iD https://orcid.org/0000-0002-3584-9982

\section{REFERENCES}

Abubakar, A., Dimitrova, R., Adams, B., Jordanov, V., \& Stefenel, D. (2013). Procedures for translating and evaluating equivalence of questionnaires for use in cross-cultural studies. Bulletin of the Transilvania University of Braşov, 6 (55), 79-86.

Adler, A. (1929). The science of living. Greenberg.

Adler, A. (1931). What life could mean to you. Hazelden.

Akçabozan, N. B., \& Sümer, Z. H. (2016). Adler yaklaşımında aile danışmanlığı [Adlerian Family Counseling]. Türk Psikolojik Danışma ve Rehberlik Dergisi, 6(46), 87-101.

Ansbacher, H., \& Ansbacher, R. (Eds.). (1956). The individual psychology of Alfred Adler. Basic Books, Inc.

Ashby, J. S., Kottman, T., \& Rice, K. G. (1998). Adlerian personality priorities: Psychological attitudinal differences. Journal of Counseling and Development, 76, 467-474. https://doi.org/10.1002/j.1556-6676.1998.tb02706.x

Ashby, J. S., Kottman, T., \& Stoltz, K. B. (2006). Multidimensional perfectionism and personality profiles. The Journal of Individual Psychology, 62, 312-323.

Brown, T. A. (2014). Confirmatory factor analysis for applied research. Guilford Publications.

Carlson, E. D. (2000). A case study in translation methodology using the health-promotion lifestyle profile II. Public Health Nursing, 17(1), 61-70. https://doi.org/10.1046/j.15251446.2000.00061.x

Carlson, J. M., \& Carlson, J. D. (2000). The application of Adlerian psychotherapy with AsianAmerican clients. Individual Psychology, 56(2), 214-225.

Carlson, J., Watts, R. E., \& Maniacci, M. (2006) Adlerian therapy: Theory and practice. American Psychological Association.

Costello, A. B., \& Osborne, J. (2005). Best practices in exploratory factor analysis: Four recommendations for getting the most from your analysis. Practical Assessment Research \& Evaluation, 10(7). https://doi.org/10.7275/jyj1-4868

Dillman Taylor, D., \& Mullen, P. R. (2019). Adlerian Personality Priority Assessment: A Psychometric Evaluation. The Journal of Individual Psychology, 75(2), 122-144.

Dillman Taylor, D., Bratton, S. C. \& Henson, R. K. (2019): Confirming the Constructs of Adlerian Personality Priority Assessment. Measurement and Evaluation in Counseling and Development. https://doi.org/10.1080/07481756.2019.1595814

Dillman Taylor, D., Gungor, A., Blount, A. J., \& Mullen, P. R. (2018). Personality Priorities and Perceived Wellness Among Counseling Trainees. The Journal of Individual Psychology, 74(2), 188-208.

Dillman Taylor, D., Ray, D. C., \& Henson, R. K. (2015). Development and factor structure of the Adlerian Personality Priority Assessment. Archives of Assessment Psychology, 5(1), 23-36.

Ergün-Başak, B., \& Ceyhan, E. (2011). Psikolojik danışma ilişkisinde Adler yaklaşımına göre cesaretlendirme [Adlerian Encouragement in Counseling Relationship]. Türk Psikolojik Danışma ve Rehberlik Dergisi, 4(35), 92-99. 
Geisinger, K. F. (1994). Cross-cultural normative assessment: Translation and adaptation issues influencing the normative interpretation of assessment instruments. Psychological Assessment, 6(4), 304-312. https://doi.org/10.1037/1040-3590.6.4.304

Gerbing, D. W., \& Hamilton, J. G. (1996). Viability of exploratory factor analysis as a precursor to confirmatory factor analysis. Structural Equation Modeling, 3, 62-72. https://doi.org/ $\underline{10.1080 / 10705519609540030}$

Hair, J. F., Black, W. C., Babin, B. J., Anderson, R. E., \& Tatham, R. L. (2010). Multivariate data analysis. Pearson.

Hambleton, R. K., Merenda, P. F., \& Spielberger, C. D. (2004). Adapting educational and psychological tests for cross-cultural assessment. Psychology Press.

Hooper, D., Coughlan, J., \& Mullen, M. (2008). Structural equation modelling: Guidelines for determining model fit. The Electronic Journal of Business Research Methods, 6(1), 53 60 .

Hu, L., \& Bentler, P. M. (1999). Cutoff Criteria for Fit Indexes in Covariance Structure Analysis: Conventional Criteria versus New Alternatives. Structural Equation Modeling, 6(1), 1-55. https://doi.org/10.1080/10705519909540118

Kefir, N. (1971). Priorities: A different approach to life style. Paper presented at the International Committee of Adlerian Summer School and Institutes (ICASSI), Tel Aviv, Israel.

Kefir, N. (1981). Impasse/priority therapy. In R. Corsini (Ed.), Handbook of innovative psychotherapies. Wiley.

Kefir, N., \& Corsini, R. J. (1974). Dispositional sets: A contribution to typology. The Journal of Individual Psychology, 30, 163-178.

Kern, R. M., Wheeler, M. S., \& Curlette, W. L. (1997). BASIS-A inventory interpretive manual: A psychological theory. TRT Associates.

Kline, R. B. (2011). Principles and practice of structural equation modeling (3rd ed.). Guilford Press.

Langenfeld, S., \& Main, F. (1983). Personality priorities: A factor analytic study. The Journal of Individual Psychology, 39, 40-51.

Mvududu, N. H., \& Sink, C. A. (2013). Factor analysis in counseling research and practice. Counseling Outcome Research and Evaluation, 4(2), 75-98. https://doi.org/10.1177/215 $\underline{0137813494766}$

Oberst, U. E. \& Stewart, A. E. (2003) Adlerian psychotherapy: An advanced approach to individual psychology. Routledge Taylor \& Francis Group.

O'Rourke, N., Psych, R., \& Hatcher, L. (2013). A step-by-step approach to using SAS for factor analysis and structural equation modeling. SAS Institute.

Pallant, J. (2010). SPSS Survival Manual, (4th ed.). McGraw Hill.

Pew, W. L. (1976). The number one priority. John's Hospital, Marriage and Family Education Center.

Sümer, Z. H., \& Rasmussen, P. R. (2012). Individual Psychology in Turkey. Journal of Individual Psychology, 68(4), 411-421.

Sweeney, T. J. (2009). adlerian counseling and psychotherapy: A practitioner's approach $\left(5^{\text {th }}\right.$ ed.). Routledge Taylor \& Francis Group.

Tabachnick, B., \& Fidell, L. (2013). Using multivariate statistics (6th ed.). Pearson.

Ward, D. E. (1979). Implications of personality priority assessment for the counseling process. Individual Psychologist, 16(2), 12-16.

Weston, R., \& Gore, P. A. J. (2006). A brief guide to structural equation modeling. Counseling Psychologist, 34(5), 719-751. https://doi.org/10.1177/0011000006286345 